\title{
Experiencia de implementación de un modelo de Atención Primaria
}

\section{Experience of implementing a primary attention model}

\author{
Myriam Ruiz-Rodríguez ${ }^{1}$, Naydú Acosta-Ramírez ${ }^{1}$, \\ Laura A. Rodríguez Villamizar ${ }^{1}$, Luz M. Uribe ${ }^{2}$, Martha León-Franco ${ }^{1}$
}

1 Departamento de Salud Pública. Universidad Industrial de Santander. Bucaramanga, Colombia.
myriam@uis.edu.co, nacostar@uis.edu.co, laurovi@uis.edu.co, marlefra@gmail.com
2 Secretaría de Salud de Santander. Bucaramanga, Colombia. luzmarinaur@hotmail.com

Recibido 2 Julio 2011/Enviado para Modificación 13 Octubre 2011/Aceptado 21 Noviembre 2011

\section{RESUMEN}

Objetivo Identificar las dificultades y los factores dinamizadores en la implementación de un modelo de atención primaria en Santander en la última década.

Métodos Estudio cualitativo con un enfoque de pluralismo y la triangulación de fuentes y actores, con análisis de crítica del límite y de los valores de los juicios (Boundary critique).

Resultados Según las categorías emergentes derivadas de la apropiación de los atributos del modelo se encuentran problemáticas tanto en aspectos filosóficoconceptuales como en la gestión operativa. No se desarrolló en la práctica el modelo planteado discursivamente en el diseño. La estrategia de APS es selectiva liderada desde el nivel departamental, centrada en acciones de predominio rural realizadas por auxiliares de enfermería y orientado al cumplimiento de metas de Salud Pública en el primer nivel de atención.

Conclusiones Se identifican dificultades tanto en el nivel nacional, departamental y municipal, que pueden ser aprovechados en otros contextos de orden nacional e internacional. Las limitantes más destacadas son de tipo estructural y determinadas por un sistema de salud de mercado de aseguramiento que opera con una lógica contractual de segmentación institucional y fragmentación operativa. Se sugieren como elementos operativos esenciales para enfrentar las problemática y fomentar la puesta en práctica de un modelo integral de APS en el país: gestión del talento humano centrado en competencias en APS, gestión local adecuada y estudios sistemáticos de evaluación.

Palabras Clave: Atención Primaria de salud, política de salud, gestión en salud, investigación cualitativa, sistemas de salud, Colombia. (fuente: DeCS, BIREME). 


\begin{abstract}
Objective Identifying barriers and dynamic factors in setting up a primary health care $(\mathrm{PHC})$ model in the Santander department during the last decade.

Methods This was a qualitative study, focusing on pluralism and triangulating sources and actors, with a critical analysis of limits and judgments values (boundary critique).

Results Philosophical/conceptual and operational management problems were found from the emergent categories related to appropriating PHC attributes. The theoretical model design was in fact not developed in practice. The PHC strategy is selective and state-led (at department level), focusing on rural interventions developed by nursing assistants and orientated towards fulfilling public health goals in the first healthcare level.

Conclusion Difficulties at national, state and local level were identified which could be useful in other national and international contexts. Structural healthcare system market barriers were the most important constraints since the model operates through the contractual logic of institutional segmentation and operational fragmentation. Human resource management focusing on skills, suitable local health management and systematic evaluation studies would thus be suggested as essential operational elements for facing the aforementioned problems and encourage an integral PHC model in Colombia.
\end{abstract}

Key Words: Primary health care, health policy, health planning, qualitative research, health care system, Colombia (source: MeSH, NLM).

$\mathrm{L}$ a atención primaria en salud (APS) desde su promulgación en Alma Ata en 1978 ha demostrado ser una estrategia efectiva para fortalecer los sistemas y servicios de salud y mejorar la calidad de vida de la población $(1,2)$. En Colombia, la APS se empezó a implementar desde 1981 (3). Sin embargo, con la promulgación de la Ley 100 de 1993 esta estrategia desapareció del escenario nacional (4). Desde el año 2004, algunos gobiernos de índole local (5) y departamental (6) han puesto en práctica en la red pública esta estrategia.

En el departamento de Santander, Colombia, en el año 2004 se puso en marcha el Modelo de Atención Integral Basado en Atención Primaria de Salud (MAPIS) fundamentado en tres pilares: la definición de Alma Ata, los atributos de la APS propuestos por Starfield (7) y los elementos de la APS integral y renovada (8). Así, la APS en Santander parte de involucrar a todos los actores del actual Sistema General de Seguridad Social en Salud-SGSSS (reorientación de actores); y retoma la regionalización 
(territorialización por provincias), con el propósito de desarrollar acciones específicas y facilitar la gestión en el amplio territorio del Departamento. Finalmente, el modelo concibe la intersectorialidad como el componente central y la participación comunitaria como el eje integrador del MAPIS.

En su estructura organizativa el MAPIS (6) se propone como un modelo de gestión de la política pública en salud para lo cual el Departamento centra su tarea hacia los municipios en: a. Gestionar la implementación del MAPIS; b. Articular el modelo entre el ámbito departamental y municipal, $y$; c. Hacer concurrencia financiera y complementariedad en el recurso humano como un incentivo para que los municipios adopten el MAPIS en sus planes territoriales de salud. Para las dos primeras tareas se definen tres equipos ubicados en los distintos niveles de gestión (6). A nivel de la Secretaría de Salud Departamental al Equipo Gestor se le asigna la función básica de articular y desarrollar actividades en pro de la gestión modelo. En el ámbito provincial, los equipos interdisciplinarios llamados Equipos Provinciales, se encargan de interactuar en los ámbitos locales y son los responsables de la articulación y fortalecimiento de los procesos gerenciales a partir de la situación de salud específica. Por último, en el ámbito municipal, se prevén Los equipos municipales encargados de liderar las acciones de intersectorialidad e integración institucional y gestionar el desarrollo de las actividades específicas de salud de competencia municipal.

En su estructura operativa, el MAPIS define que la puerta de entrada al sistema está determinada por auxiliares de enfermería con asignación de familias; ellas canalizan a los usuarios a los servicios de salud facilitando el acceso, disminuyendo barreras geográficas, económicas, organizativas y administrativas e incentivando el uso de los servicios (6). La caracterización de las familias y sus necesidades se convierten en el sustrato para desencadenar procesos de planeación que movilicen recursos de todos los sectores municipales en pro de la solución de los problemas priorizados.

No obstante, la trayectoria del MAPIS, la inversión de recursos y su relevancia para la política de salud, este modelo no ha sido evaluado en forma sistemática. El propósito de este artículo es presentar los resultados de la experiencia de Santander en términos de las categorías emergentes derivadas de la apropiación de los atributos del MAPIS, con el fin de identificar las dificultades y los factores dinamizadores para su desarrollo 
que sirvan como lecciones para las autoridades de salud del ámbito nacional e internacional que se encuentran implementando la APS como eje articulador del sistema de salud.

\section{METODOS}

Este trabajo hace parte de un proyecto macro que determinó el enfoque del diseño y las características de la gestión e implementación de la APS en Bogotá y Santander mediante un estudio de triangulación de métodos de investigación. Los resultados que se presentan en este artículo provienen de la perspectiva cualitativa de dicho estudio con triangulación de fuentes y actores, con análisis de crítica del límite y de los valores de los juicios (Boundary critique) (9), por medio del estudio de casos de seis municipios seleccionados por criterios de cobertura del MAPIS, condiciones de ruralidad, necesidades básicas insatisfechas y representatividad por provincias. Así, se estudiaron tres municipios con un grado de desarrollo mayor del MAPIS y otros tres menos desarrollados (Tabla 1). La recolección de la información se realizó en el año 2010.

Tabla 1. Características de los municipios participantes en el estudio

\begin{tabular}{ccccc}
\hline Municipio & Población & $\begin{array}{c}\text { Número de } \\
\text { veredas }\end{array}$ & $\begin{array}{c}\% \\
\text { Población } \\
\text { rural }\end{array}$ & $\begin{array}{c}\% \\
\text { NBI }\end{array}$ \\
\hline Mayor desarrollo MAPIS ${ }^{\text {a }}$ & 18714 & 64 & 88,3 & 51,1 \\
1 & 3845 & 9 & 67,8 & 25,6 \\
2 & 11258 & 25 & 56,1 & 41,9 \\
3 & & & & \\
Menor desarrollo MAPIS & & 12 & 18,6 & 20,9 \\
4 & 18581 & 36 & 79,8 & 30,4 \\
5 & 5566 & 72 & 75,0 & 48,8 \\
\hline 6 & 15276 & & & \\
\hline
\end{tabular}

El estudio siguió tres fases. Una primera, de análisis documental para identificar el contexto sociopolítico, el marco programático y contractual de acciones del modelo y el contenido valorativo de principios y racionalidad política del MAPIS, así como los atributos del modelo: integralidad, intersectorialidad, participación comunitaria, longitudinalidad (vínculo), primer contacto (accesibilidad con puerta de entrada definida al sistema), coordinación, reorientación de actores del SGSSS y territorialización. 
En la segunda fase, se entrevistaron funcionarios de la Administración Municipal y funcionarios de salud con cargos directivos y operativos para explorar el enfoque, el modelo de gestión y las intervenciones aplicadas en el contexto de MAPIS. De otro lado, se realizaron grupos focales con miembros de la comunidad beneficiaria, para vislumbrar formas locales de apropiación, interpretación y crítica a las intervenciones desarrolladas a partir del MAPIS. Finalmente, y de manera simultánea a las entrevistas, se realizaron visitas de observación participante a los municipios para conocer las dinámicas de relación e interacción de las intervenciones del MAPIS y establecer el alcance de participación comunitaria.

En la tercera fase, se realizaron talleres de devolución de los resultados con participantes de los distintos niveles de gestión del MAPIS acorde con el enfoque de Boundary critique, para propiciar entre los involucrados el debate e innovación organizacional en el marco de los procesos generados por el MAPIS.

Para el análisis de la información se identificaron como categorías a priori los atributos del modelo y para identificar las categorías emergentes se siguió el procedimiento de codificación y categorización propuesto por la teoría fundamentada (10) y se utilizó el software Atlas Ti para apoyar tal procedimiento. El estudio fue aprobado por los Comités de Ética de las instituciones académicas que participaron el proyecto macro (Pontificia Universidad Javeriana y Universidad Industrial de Santander).

\section{RESULTADOS}

A partir de la triangulación de fuentes y actores se pudo encontrar puntos convergentes y divergentes, en cada municipio y entre municipios, en cuestión de planeación, ejecución y apropiación de los atributos del MAPIS, lo cual permitió identificar cinco temas principales:

Acompañamiento del equipo provincial y del profesional de enlace

Los funcionarios entrevistados en los municipios no hablaron de los equipos provinciales, ni de los profesionales de enlace, quienes lideran dichos equipos, hasta que se les interrogó de manera explícita sobre su interrelación con estos. Fueron recurrentes los discursos que hicieron referencia al papel de vigilancia, veeduría y cumplimiento de las acciones 
del Plan de Intervenciones Colectivas (PIC). Escasearon los discursos referidos a asesoría, orientación y abogacía del equipo provincial/ profesional de enlace y fueron recurrentes los relatos referidos al rol de capacitadores en temas de salud, pero no en su papel de acompañamiento del MAPIS: “ ...vino un doctor, pero él lo que venía era a dictar charlas de enfermedades de transmisión sexual, ,[...], pero que diga vamos a trabajar a jalonar un programa, un proceso, que lo orienten a uno por lo menos en esto de APS...que yo vea que el equipo provincial me oriente como es eso, para qué es, ó para dónde vamos, ino!" (Enfermera Empresa Social del Estado -ESE).

Otro tipo de discurso recurrente entre los Secretarios de Salud y Alcaldes reconoce el papel de acompañamiento que el profesional de enlace hace, no al desarrollo del MAPIS, sino en el campo de salud pública, especialmente en lo que tiene que ver con la programación de los ejes del Plan Nacional de Salud Pública.

Estos discursos pueden ser explicados por algunos de los hallazgos del análisis documental: a) el origen de la financiación de dichos cargos provienen del rubro de salud pública, por lo tanto le son asignadas otras tareas distintas al acompañamiento del MAPIS, en especial las del PIC y b) la falta de continuidad del equipo en la cabecera de provincia debido a que su contratación (excepto el del profesional de enlace) no es de planta, por tanto, su vinculación y continuidad se ve afectada por la dinámica de contratación en el sector público.

Proceso de identificación de necesidades

Operativamente, en su diseño el MAPIS indica la aplicación de la ficha familiar, complementada con el ecomapa y el familiograma, para caracterizar la población e identificar sus necesidades, guardando un enfoque familiar y comunitario, de tal manera que se desencadene una serie de acciones intersectoriales y con participación comunitaria.

Se encontró que la aplicación de fichas no incluyó ecomapas ni familiogramas y alcanzó coberturas progresivas hasta lograr el $100 \%$ del área rural y urbana en los municipios 1,2 y 4, y en algunas veredas de los municipios restantes. No obstante la información recolectada en los seis municipios, ésta solo fue usada en el municipio 1 para intervenir las familias en riesgo, y en el 2 para la focalización de subsidios de mejoramiento de 
vivienda. No se identifican discursos que señalen cómo el uso de dicha información permitió promover la movilización de recursos y actores alrededor de las necesidades identificadas.

A partir de la voz de los entrevistados se pudo reconocer que el uso de la información recolectada con la ficha familiar se limitó por: a. Desconocimiento del manejo del software estipulado por la Secretaría de Salud para sistematizar la información; b. Falta de personal específico para la digitación y consolidación de la información; c) falta de seguimiento y evaluación de la información recolectada por actores diferentes a la auxiliar. El siguiente testimonio da cuenta de ello: "Yo decía, ¿qué hago con estas fichas?, las aplico y ¿qué?. Entonces yo le pregunté a la doctora y me dijo que tocaba pasarlas a un programa. Yo las guardé, y como soporte del PIC, las primeras fichas las digité." (Enfermera ESE).

Al triangular información del análisis documental, visita de campo y entrevistas pudimos identificar cómo el alcance que la auxiliar da a los resultados de la aplicación de la ficha está condicionado por una lógica contractual orientada al cumplimiento de metas de contratos, en un sistema de salud en el que las acciones a realizar en la población están definidas taxativamente en los planes de beneficios sin dar cabida a procesos de planeación y movilización de actores y recursos frente a problemas de salud, como lo que propone el MAPIS.

Reorientación de actores institucionales del SGSSS en el ámbito local

En general, en los discursos de los actores institucionales se identifica que estos no hicieron ajustes para reorganizar los servicios acorde con los atributos de MAPIS. Las enfermeras y gerentes de las ESE hablaron del papel pasivo, colaborativo y enfocado a demanda inducida de las Empresas Promotoras de Salud (EPS). Algunas expresiones como "ellas colaboran" "ellas ayudan" se encontraron de manera recurrente en los discursos. De otro lado, estos actores también señalan la falta de reconocimiento que las EPS tienen, no solamente acerca del MAPIS, sino de las responsabilidades en cuanto a las acciones de $\mathrm{P}$ y $\mathrm{P}$ y hacen notar cómo las funciones que normativamente tienen las EPS impiden que se puedan desarrollar iniciativas distintas a las estipuladas en los planes de beneficios. 
Los Secretarios de Salud, en general perciben que a pesar de los esfuerzos de articulación con la ESE, el rol de las instituciones se sigue circunscribiendo al marco de las responsabilidades estipuladas normativamente y no se dan cambios en cuanto al modelo de prestación, al de atención y a la forma de trabajar integradamente en pro de la APS. En la mayoría de los casos, estos esfuerzos son resultado de esfuerzos individuales, de habilidades personales o gerenciales.

Los funcionarios de las EPS reconocen en forma categórica que su papel en el modelo se refiere a la ejecución de acciones de demanda inducida a programas y al seguimiento de pacientes: "...se contrata una persona para promover los programas que existen y pues para en dado caso que se presente algún evento centinela que nosotros llamamos, entonces ellos se le haga seguimiento al paciente..." (Gestor EPS). También se percibe en el discurso de estos actores el énfasis que ponen en estipular que su función está referida a la prestación del Plan Obligatorio de Salud (POS).

Adicionalmente, varios Secretarios de Salud identificaron que el hecho de que la APS no esté en la normatividad es el obstáculo más importante para desarrollar servicios basados en APS: “...me gustaría que el modelo de atención primaria fuera obligatorio, ¿si? es decir, no tanto obligatorio, sino que estuviera dentro de una normatividad en la que los municipios deben implementarla." (Secretario de Salud).

En general, pudo identificarse en los discursos cómo las segmentación de los planes de atención impiden que el modelo pueda desarrollarse tal como está concebido y cómo la garantía de estas acciones se da por el cumplimiento de contratos y no por resultados en los indicadores de salud: "...es importante recalcar que con el hospital tenemos contratado lo que es el eje programático del plan territorial de salud pública y también lo que es la prestación de servicio. De hecho una manera de nosotros garantizar que esto se organiza es mediante la contratación" (Secretario de Salud)

Vínculo entre el equipo de salud del modelo y la comunidad

Aunque en cinco de los seis municipios existía una sola institución pública de atención, no se encontraron discursos, ni por parte de los funcionarios, ni de la comunidad, que trascendiera la atención centrada en la consulta. En ninguno de los municipios se conformó el equipo de salud municipal para el MAPIS por lo tanto, el modelo es muy débil en cuanto a la creación 
de vínculos entre la atención individual por parte de profesionales de salud y las familias y comunidades. En general, el vínculo que se creó en los seis municipios se redujo a la relación entre las familias y las auxiliares de enfermería para el área rural, sin tener cobertura para todas las veredas de cada municipio.

Precisamente, al respecto, muchas de las respuestas de los entrevistados señalaron varias condiciones que impidieron que esta relación fuera continua en el tiempo. En cinco de los seis municipios no hubo continuidad de auxiliares, por problemas en la contratación. Algunos municipios enfrentaron serias dificultades para conseguir auxiliares que desearan ir a trabajar a pequeños municipios o al área rural.

El gerente de la ESE del municipio 1 relató su experiencia para mantener el recurso de auxiliares en su municipio resaltando cómo distintos factores relacionados con la normatividad en la contratación y con la falta de incentivos atentan contra el firme deseo de mantener un equipo que pueda desarrollar una relación estable y a largo plazo: “....eso de la contratación de personal afecta mucho...nosotros contratábamos las auxiliares...por doce meses y se liquidaban...la contraloría nos pasó un requerimiento de que no se podía...porque eso era fraccionamiento de contrato y que teníamos que contratar por Cooperativa...Nos tocó hacer un esfuerzo como empresa, y hacerle caso a la Contraloría y contratar una Cooperativa.... Nos tocó asumir, además del sueldo, un incentivo a final de año... para motivar al empleado, claro que la empresa tiene un gasto más grande, es muy duro, pero las mismas Leyes a veces también van en contracorriente contra nosotros" (Gerente ESE).

De otro lado, pudo identificarse en los grupos focales con comunidad, que las auxiliares que llevaban mucho tiempo trabajando con la comunidad, que estaban capacitadas en el trabajo en el marco de APS y que residían permanentemente en la misma vereda, eran calificadas como las de mejor desempeño en el trabajo comunitario alrededor de las necesidades de salud de la misma.

Imaginario de la APS y su relación con el quehacer cotidiano

Se identificaron dos tipos de discursos, los cuales estuvieron relacionados principalmente con el grado de desarrollo y apropiación del MAPIS. Un primer discurso de los funcionarios del municipio $1 \mathrm{y}$ de una vereda del municipio 6 describen a la APS como una estrategia articuladora del desarrollo de la población. Es importante remarcar, cómo a partir del 
análisis documental pudimos identificar algunas condiciones que pueden ayudar a comprender estos discursos: los funcionarios de esos municipios son los que tienen mayor continuidad en la aplicación de la estrategia de APS (incluso, mucho antes de adoptar el MAPIS), y en el caso particular del municipio 1 , han recibido más capacitación y monitoreo por parte del departamento y educación continua en APS por parte de los directivos de la ESE.

Un segundo tipo de discurso, visualiza a la APS de manera selectiva y limitada siendo las palabras más frecuentes para definirla: "trabajo extramural", "nivel básico de atención", "programas de PIC y de Prevención y Promoción-PyP", "demanda inducida" y "aplicación de fichas domiciliarias". En el análisis documental pudieron identificarse varias situaciones que pueden estar explicando estos testimonios: a. La normatividad del SGSSS no contempla la APS como un eje articulador del sistema colombiano; b. Existe discrecionalidad por parte de las autoridades municipales para adoptar el MAPIS, y; c. El fuerte énfasis que MAPIS tiene hacia las acciones extramurales circunscritas al ámbito de la salud pública.

\section{DISCUSIÓN}

Los resultados muestran que existe una disonancia entre el modelo diseñado y planteado en el departamento y la operación municipal que surge en la práctica. Así, contrario a lo que se planteó discursivamente en el diseño como un modelo de gestión integral en salud (6), en la práctica operó como una estrategia de APS selectiva liderada desde el nivel departamental, que se centra en acciones de predominio rural realizadas discontinuamente por auxiliares de enfermería y orientado al cumplimiento de metas de Salud Pública (PIC y PyP) en el primer nivel de atención.

Los hallazgos permiten identificar dificultades en diversos niveles para el desarrollo de la APS. En el nivel nacional estarían factores de la estructura del SGSSS, la ausencia de normatividad sobre la operación de la APS y la política de flexibilidad laboral. En el nivel departamental las dificultades están relacionadas con la territorialización incompleta y los mecanismos de seguimiento y evaluación orientados al cumplimiento de metas del PIC, y por último, en el nivel municipal se destacan limitantes como la operación centrada en la auxiliar de enfermería, la falta de articulación entre aseguradores y prestadores alrededor de la APS y la necesidad de recurso humano con formación en APS y estabilidad laboral. 
La mayoría de las dificultades son de tipo estructural y están determinadas por un sistema de salud, que en Colombia tiene multiplicidad de actores, y opera con una lógica contractual con segmentación institucional y fragmentación operativa que no está orientado a la solución de problemas de la población sino a la aplicación de planes y el cumplimiento de metas, con dilución de responsabilidades para la atención salud. Estas características también han sido identificadas por otros autores como problemáticas para la atención en salud en sistemas de salud basados en mercado de aseguramiento y obstaculiza la implementación de estrategias integrales como las que propone un modelo de atención integral basado en APS (11-15).

Como consecuencia de estas dificultades estructurales, los municipios con mayor desarrollo de la APS debieron realizar esfuerzos adicionales con el fin de superarlas, especialmente las relacionadas con la fragmentación de acciones, la centralización y estandarización de las prioridades de salud pública y los modelos hegemónicos de contratación pública.

Otro factor estructural identificado, hace referencia al recurso humano, en el que convergen aspectos como la irregularidad en su contratación, la falta de incentivos para el trabajo en zonas alejadas y la falta de formación de los profesionales con un enfoque que trascienda del biomédico e individual a uno familiar y comunitario.

En el momento actual en el que Colombia recién acaba de dictar la Ley 1438 de 2011, que incluye a la APS como la estrategia estructurante para la atención en salud y define la organización de equipos básicos, el ente rector de la salud en el país debería considerar algunos elementos operativos que, de acuerdo a los resultados de esta investigación se configuran como esenciales para la puesta en práctica de la APS en los territorios: (a) Asignación de equipos multidisciplinarios estables y capacitados en las competencias en APS; (b) Desarrollo de mecanismos y procesos de concertación que integren a los diferentes actores del SGSSS alrededor de las necesidades y los planes de desarrollo locales; y (c) Monitoreo y evaluación con sistemas de información sencillos y unificados que incluyan el seguimiento de los procesos de intervención y los cambios en los indicadores de salud, además de estudios sistemáticos de evaluación que en otros contextos han permitido identificar áreas de mejoramiento en la aplicación de la APS $(16,17)$ • 
Agradecimientos: A Colciencias (contrato RC 683 de 2009), la Secretaría de Salud de Santander (contrato 01905 de 2009) y la Universidad Industrial de Santander (registro VIE 6868-0154-13). A Angélica María Amado Niño por el apoyo en la organización de la información y redacción de textos. A Fanny Ochoa Camargo y Cristina Ramírez Jaimes por el apoyo en el operativo de campo de recolección de información. A funcionarios de los municipios y población que aportaron información para la investigación.

Conflicto de intereses: Ninguno

\section{REFERENCIAS}

1. Organización Mundial de la Salud. La atención Primaria de Salud, Más Necesaria que Nunca. Informe sobre la salud en el mundo 2008. Ginebra: OMS, 2008.

2. Starfield B, Shi L, Macincko J. Contribution of Primary Care to Health Systems and Health. Milbank Q. 2005; 83(3):457-502.

3. Ministerio de Salud. Informe al Honorable Congreso de la República. Bogotá: MS- Instituto Nacional de Salud, 1981.

4. Organización Panamericana de la Salud. La organización Panamericana de la Salud y el Estado Colombiano. Cien años de historia 1902-2002. Bogotá: OPS, 2002.

5. Vega R, Acosta N, Mosquera PA, Restrepo MO. La política de salud en Bogotá, 2004-2008. Análisis de la experiencia de atención primaria integral de salud. Revista medicina social 2008; 3(2):148-169.

6. León MH, Ramírez AN, Uribe LM. Modelo de Atención Basado en Atención Primaria de Salud. Experiencia en Santander. Revista del Observatorio de Salud Pública de Santander 2007; 3(1):32-38.

7. Starfield B. Atención Primaria: equilibrio entre necesidades de salud, servicios y tecnologías. Barcelona: Masson, 2001.

8. Macinko J, Montenegro H, Nebot AC, Etienne C. La renovación de la atención primaria de salud en las Américas. Rev Panam Salud Pública. 2007; 21(2/3):73-84

9. Midgley G. Systemic Intervention: Philosophy, Methodology, and Practice. New York: Kluwer Academic/Plenum Publishers, 2000.

10. Strauss A, Corbin J. Bases de la Investigación Cualitativa. Técnicas y Procedimientos para Desarrollar la Teoría Fundamentada. Medellín: Editorial Universidad de Antioquia, 2002. p.219-231.

11. Vargas I, Vázquez ML, Mogollon AS, Unger JP. Barriers of access to care in a managed competition model: lessons from Colombia. BMC Health Serv Res 2010; 10:297.

12. Gold M. Beyond coverage and supply: measuring access to healthcare in today's market. Health Serv Res 1998, 33:625-652.

13. Guerrero R. El Concepto de la Protección Social. Cuadernos de protección social No. 3. Bogotá: Ministerio de la Protección Social, 2006.

14. Hsiao WC. "Marketization"-The Illusory Magic Pill. Health Economics 1994; 3(6): 351-357.

15. Ruiz-Rodriguez M, Wirtz V, Nigenda G. Organizational elements of health service related to a reduction in maternal mortality: the cases of Chile and Colombia. Health Policy 2009; 90(2):149-155.

16. Broemeling A, Watson D, Black Ch, Reid R. Measuring the Performance of Primary Health Care. Vancouver: Centre for Health Services and Policy Research. University of British Columbia, 2006.

17. Macinko J, Almeida C, Klingelhoefer DSP. A rapid assessment methodology for the evaluation of primary care organization and performance in Brazil. Health Policy Plan. 2007; 22(3):167-177. 